\title{
Additional information and clarifications surrounding the Many dimensions interpretation of quantum mechanics, and a new thought experiment
}

Keywords:

Decoherence, Quantum Tunneling, Double slit experiment.

John Taylor

Contents:

1. Why it is that according to this interpretation the Schrodinger equation is incomplete

2. Nature of the wave function

3. Quantum tunneling

4. Double slit experiment

5. New variation of the double slit experiment

\subsection{Abstract:}

This is an extension of my previous pre print, in which it will be again once iterated the clarifications of the many dimensions interpretation. This article was published on OSF preprints, titled 'Additional information and clarifications surrounding the Many dimensions interpretation of quantum mechanics'(https://osf.io/xq8jm). There will be information included from that article, as well as an extension of a new thought experiment in quantum mechanics, which will reach various philosophical implications. This will not be fully developed and there are plans for a more refined version.

\subsubsection{Introduction}

Throughout this article we will revisit the many dimensions interpretation of quantum mechanics, with much more clarity. It is a revisit to my previous article published on OSF preprints, titled 'Many dimensions and thought experiment in Quantum mechanics'. Furthermore we shall briefly outline a new variation of the double slit experiment.

Why it is that according to this interpretation the Schrodinger equation is incomplete. According to this interpretation of quantum mechanics a particle exists physically as a spread out wave, in which most of it exists in more than three dimensions, but there is typically however, one point that exists in just three dimensions, and this is the part that we observe, and when observed it starts to behave as a particle, and the probability of finding this point is described by the wave function. The Schrodinger wave equation is therefore incomplete because it is describing the particle in terms of existing in three dimensions, when in fact most of it exists in more than three dimensions. Therefore it is inaccurate 
because it is describing particles, which actually exist mainly in more than three dimensions as existing in three dimensions, according to this interpretation.

Nature of the wave function The wave function does not tell you the probability of finding the particle in a certain location, but the probability of finding the part of this spread out wave that exists in three dimensions, in a certain location. When the wave function has collapsed to an eigenstate, it can then be confirmed that the other possibilities that were not observed of the wave function have manifested in more than three dimensions.

\section{Quantum tunneling:}

Quantum tunneling occurs, because there is a probability of detecting the point of the Schrodinger wave that exists in three dimensions on the other side of the potential barrier, not the probability of detecting the whole particle (which exists as a spread out wave). The $3 \mathrm{~d}$ part of this spread out wave is able to penetrate the potential barrier classically, because occasionally it might have energy to do so for various reasons, such as an electron knocking into an electron, could give it more kinetic energy to do so. The probability of these occurring decreases exponentially as the width of the barrier increases, and is a similar explanation to that of Bohmian Mechanics.

\section{Double slit experiment}

1) When an electron is fired out of an electron gun it is travelling as a wave in which most of it is travelling in more than three dimensions, but typically one point is travelling in just three dimensions (the point that we measure).

2) When it encounters the double slit the waves interfere with each other across dimensions, and an interference pattern builds up, over time as the electrons hit the detector screen one by one. The interference pattern can be explained through constructive and destructive interference. We cannot observe the particle as a wave because most of this wave exists in more than three dimensions (for example a part could exist in 7 dimensions), and since we are only capable of detecting objects that exist in three dimensions, we will only be able to detect the part of the Schrodinger wave that exists in three dimensions, nothing else and from this it can be concluded that we cannot observe it as a wave, because when the part that exists in three dimensions is measured it starts to behave like a particle, and loses it's wave like properties. Furthermore we cannot even observe the parts of the wave that exist in more than three dimensions, in just three dimensions anyway because in order to observe something it needs to be observed in it's entirety, and therefore needs be observed in all of it's dimensions. For example you cannot observe a 3 dimensional object in just 1 dimension, however you can measure these properties individually, for example measuring the length or width of a particular object.

3) The collapse of the wave function occurs when the part of the wave function that exists in three dimensions is observed and thus isolated so that it now behaves as a particle, and a more detailed explanation of this mechanism is explained in the previous article.

5 New variation of the double slit experiment

This experiment imagines a scenario in which flies are placed in a box, and there are two slits in the box on the other side, there is a detector which would have some food to attract 
the flies over. The flies would be placed in one at a time. Some how the box would be in a state in which there would be no decoherence. Eventually as you detect the flies coming through you would expect to get an interference pattern.

This experiment if achieved, would be able to tell you about the nature of observation. You could draw the conclusion (after repeating it with multiple living organisms), that observers either can or cannot observe themselves depending on whether you get an interference pattern. In addition there are also some philosophical conclusions which could be drawn from this, being that the fly made the conscious decision to go through each slit and an interference pattern was produced, it would suggest that our conscious decisions are not really true decisions, because if the fly really was able to decide which slit to go through then it could have easily decided to go through the slit and not make an interference pattern, but if you did consistently observe this(interference pattern) then you would have to conclude that there was no real decision being made here at all since the fly was under the same physical laws as all objects set in motion, and could not evade producing an interference pattern. Additionally the opposite result could be concluded about our conscious decisions.

\section{Conclusion:}

This here therefore brings more clarity and a more detailed explanation to points in my previous article, which were poorly explained. Furthermore a new thought experiment has been briefly explored, which will need development but is on its way, in the next paper. There will be further emphasis on the philosophical conclusions, which can be drawn from such a thought experiment.

\section{References:}

1) Taylor, John j., 2018. "Additional Information and Clarifications Surrounding the Many Dimensions Interpretation of Quantum Mechanics". Open Science Framework. February 16. doi:10.17605/OSF.IO/XQ8JM.

2)Taylor, John j., 2018. "Additional Information and Clarifications Surrounding the Many Dimensions Interpretation of Quantum Mechanics". Open Science Framework. February 16. doi:10.17605/OSF.IO/XQ8JM 